\title{
Intra-articular proximal jig pin placement during tibial plateau leveling osteotomy
}

\author{
Jacqueline V.J. Cavalcanti ${ }^{1}$, Stanley E. $\mathrm{Kim}^{2 *}$ and James Colee ${ }^{3}$ \\ ${ }^{1}$ Department of Veterinary Clinical Medicine, College of Veterinary Medicine, University of Illinois, Urbana, \\ IL 61802, USA \\ ${ }^{2}$ Department of Small Animal Clinical Sciences, College of Veterinary Medicine, University of Florida, Gainesville, \\ FL 32610, USA \\ ${ }^{3}$ Department of Statistics, Institute of Food and Agricultural Sciences, University of Florida, Gainesville, FL 32611, USA
}

\begin{abstract}
Background: During tibial plateau leveling osteotomy (TPLO), a TPLO jig is often used. For placement of the jig, one of the pins is placed slightly distal to the joint line. Erroneous pin placement may lead to intra-articular damage; however, the path of the pin tract has not been thoroughly investigated.

Aim: To document the rate and identify potential risk factors of intra-articular jig pin placement in dogs undergoing TPLO with the use of a TPLO jig.

Methods: Medical records and pre- and postoperative radiographs (2007-2017) of 696 dogs with TPLO performed with a jig were reviewed. Primary surgeon and tibial plateau angles (TPA) were recorded. Postoperative radiographs were evaluated and classified according to intra-articular jig pin placement. Medial tibial plateau jig pin placement was defined as a radiolucent tract on the osteochondral junction of the medial tibial plateau. Lateral tibial plateau placement was defined as a radiolucent tract within $3 \mathrm{~mm}$ of the medial tibial plateau with a pin trajectory penetrating the lateral tibial plateau. Rates of intra-articular jig pin placement were calculated, and associations between intra-articular jig pin placement and surgeon experience and TPA were assessed with a chi-squared test.

Results: Thirty-seven (5.32\%) dogs had intra-articular placement of the jig pin. Seven dogs had medial tibial plateau jig placement, and 30 had lateral tibial plateau placement. There was no relationship between the TPA or surgeon level of experience and intra-articular placement of the pin.

Conclusion: This study serves as a reminder to be cautious when placing the proximal jig pin during TPLO to avoid intra-articular placement. In addition, guidelines for evaluating proximal jig pin placement on postoperative radiographs are provided.
\end{abstract}

Keywords: Jig, Tibial plateau angle, Tibial plateau leveling osteotomy.

\section{Introduction}

The tibial plateau leveling osteotomy (TPLO) is one of the most commonly performed surgical treatments for dogs with cranial cruciate ligament rupture (Duerr et al., 2014). The procedure involves creating a radial osteotomy of the proximal tibia, rotation of the tibial plateau segment, and rigid stabilization with internal fixation (Slocum and Slocum, 1993). Use of a jig, which attaches to the proximal and distal tibia using bone pins, was designed to aid in osteotomy orientation, to stabilize the bone segments during osteotomy rotation and reduction, and to facilitate limb alignment (Devine et al., 2003). Intraoperatively, the location of the stifle joint line is typically identified with a hypodermic needle, and the proximal bone pin for the jig is placed approximately $3 \mathrm{~mm}$ distal to the articular surface, immediately caudal to the medial collateral ligament (Schmerbach et al., 2007). Given the close proximity of this pin to the joint, there is a concern that malpositioning and/or malorientation could lead to entry into the articular space, causing damage to the cartilage and/or menisci. To the best of our knowledge, radiographic evidence of intra-articular jig pin placement has not been well investigated.

The purpose of this study is to estimate and report the incidence of intra-articular jig pin placement by evaluating the position of the proximal jig pin hole in dogs that underwent TPLO with use of a TPLO jig, and to identify possible risk factors for intraarticular jig pin placement. We hypothesized that a low experience level of the primary surgeon and tibial plateau angle (TPA) higher than $30^{\circ}$ would be associated with intra-articular jig pin placement.

\section{Inclusion criteria}

\section{Material and Methods}

Radiographs and medical records (January 2007-July 2017) for all dogs that underwent TPLO using a jig for

*Corresponding Author: Stanley E. Kim. Department of Small Animal Clinical Sciences, College of Veterinary Medicine, University of Florida, Gainesville, FL 32610, USA. Email: stankim@ufl.edu 
cranial cruciate ligament rupture at the University of Florida Veterinary Teaching Hospital were reviewed retrospectively. Records were included in the study only if both mediolateral and caudocranial projection radiographs of the tibia, including the stifle and hock joints, were obtained prior to and immediately following the surgery of all dogs.

\section{Data collection}

The experience of the primary surgeon was recorded as either board-certified surgeon, second-year resident, or third-year resident. Because the first-year residents do not perform orthopedic surgeries as a primary surgeon at our hospital, all surgeries logged with the first-year resident were considered to have been performed by faculty. The level of experience for a board-certified surgeon was not further subclassified.

Preoperative radiographs were evaluated and the TPA was measured using a conventional method (Dismukes et al., 2008) by one observer (J.V.J.C.). Postoperative radiographs were evaluated for the location of placement of the proximal jig pins based on the location and trajectory of the radiographically evident pin tract. The shortest distance from the proximal jig pin hole to the osteochondral junction of the tibial plateau on the mediolateral projection radiographs was measured. The pin hole tract was also reviewed on the caudocranial views. Pin placement was considered intra-articular if the pin tract penetrated the osteochondral junction on either radiographic view. The erroneous placement was classified according to the location of the intra-articular jig pin placement as a medial or lateral plateau. Medial plateau intra-articular jig pin placement was defined as a visible radiolucent tract directly over and through the osteochondral junction of the medial tibial plateau in the mediolateral view (Fig. 1A and B). Lateral plateau intra-articular jig pin placement was defined as a radiolucent tract within $3 \mathrm{~mm}$ of the medial subchondral bone surface on the mediolateral view (Fig. 2A), and jig pin trajectory penetrating the osteochondral junction
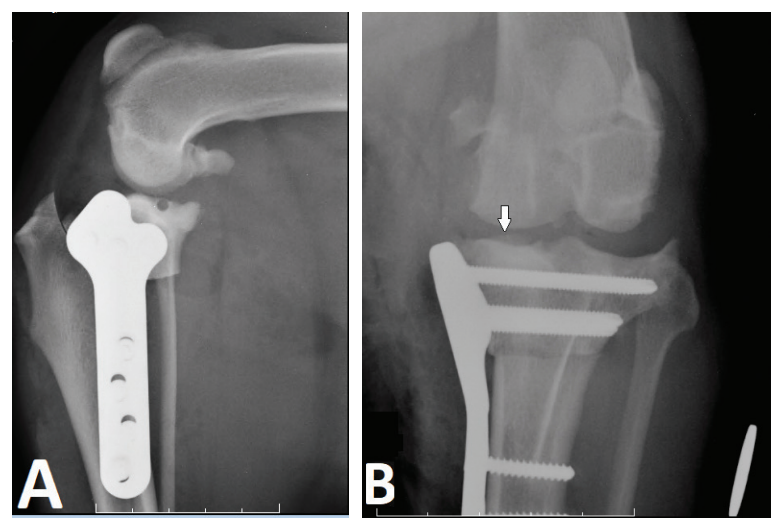

Fig. 1. Mediolateral (A) and craniocaudal (B) radiographs illustrate medial tibial intra-articular jig pin hole placement. The white arrow indicates an osteochondral fragment created during intra-articular pin placement. of the lateral tibial plateau on the caudocranial view (Fig. 2B). For those dogs that had evidence of intraarticular jig pin placement, details such as age, sex, breed, and weight were recorded.

\section{Statistical analysis}

A chi-squared test was performed to test if there was an association between intra-articular jig pin placement and level of experience of the surgeon, according to residency year and board-certified surgeon status. The Cochran Armitage trend test was also performed to test if an increase in experience decreased the odds of intra-articular jig pin placement. A chi-squared test was performed to test for any association between TPA (lower versus higher than $30^{\circ}$ ) and intra-articular jig pin placement. $p$ value $<0.05$ was considered significant.

\section{Results}

A total of 1150 records were evaluated; 696 stifles met the inclusion criteria. The distribution of radiographs by side was uniform; $365(52.5 \%)$ dogs had TPLO of the left hind limb, and 331 (47.5\%) dogs had TPLO of the right hind limb. The mean \pm standard deviation preoperative TPA was $27.5^{\circ} \pm 3.5^{\circ}$. The TPA was $\leq 30^{\circ}$ in $552(79.3 \%)$ dogs, and $>30^{\circ}$ in $144(20.7 \%)$.

Intra-articular placement of the temporary jig pins was identified in $37(5.32 \%)$ stifles according to the position of the radiolucent pin tract observed on the postoperative radiographs. Seven $(19 \%)$ were classified as medial tibial plateau jig pin placement, and 30 dogs $(81 \%)$ were considered as lateral tibial plateau jig placement. The TPA was $\leq 30^{\circ}$ in $30(81 \%)$ dogs, and $>30^{\circ}$ in 7 $(19 \%)$ dogs with intra-articular jig placement. There was no association between the TPA and intra-articular placement of the jig pin $(p=0.79)$. The predominant breed of dogs with intra-articular jig pin placement was Labrador Retriever $(n=12)$, followed by mixed breed dogs $(n=10)$, German Shepherd $(n=4)$, Rottweiler $(n=3)$, and Weimaraner $(n=2)$. The median age was 6 years (range: $3-12$ years). There were 15 castrated
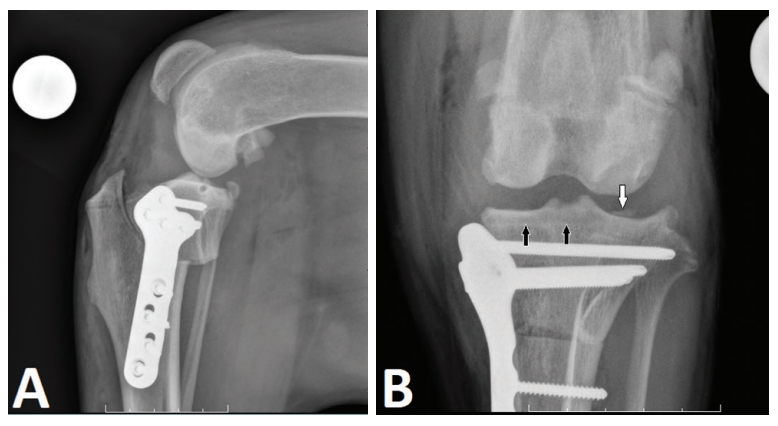

Fig. 2. Mediolateral (A) and craniocaudal (B) radiographs illustrate lateral tibial intra-articular jig pin hole placement. The black arrows show the pin tract distal to the medial subchondral bone, and the white arrow shows an osteochondral fragment created during intra-articular pin placement. 
males and 22 spayed females. The median body weight was $31.5 \mathrm{~kg}$ (range: $6.8-69.7 \mathrm{~kg}$ ).

Of the cases that had intra-articular jig pin placement, TPLO was performed by board-certified surgeons in 15 $(40.5 \%)$ stifles, by second-year residents in $10(27 \%)$ stifles, and by third-year residents in 12 (32.5\%) stifles. There was no association between intra-articular jig pin placement and experience level of the primary surgeon $(p=0.4)$.

\section{Discussion}

This retrospective study reports a 5.3\% incidence of intra-articular placement of the proximal jig pin in dogs that underwent TPLO. While the placement of the jig pin is temporary, the trauma caused by intra-articular pin placement may have consequences. Suboptimal clinical outcomes following TPLO may be contributed by articular cartilage damage and/or potential meniscal injury. In addition, intra-articular pins may cause intra-articular fractures. Unfortunately, we were not able to investigate the effects of intra-articular jig pin positioning on overall outcomes with our study design; thus, whether the patterns of injury we identified have clinically relevant consequences remains unknown.

Postoperative meniscal damage is reported to occur after TPLO, (Fitzpatrick and Solano, 2010; Pacchiana et al., 2003; Thieman et al., 2008) but the cause seems to be unclear (Thieman et al., 2008). It has been recommended to use the insertion of a 25 -gauge needle immediately caudal to the medial collateral ligament in the proximal-most aspect of the tibia as a guide for the proximal jig pin placement (Devine et al., 2003; Kowaleski et al., 2012). A prospective study in 40 canine cadaveric stifles reported grossly visible iatrogenic medial meniscal damage from a medially placed hypodermic needle in dogs undergoing TPLO (O'Brien and Martinez, 2009). In that study, tibial articular cartilage and medial meniscal damage occurred in up to $80 \%$ of stifles; the needles $(20 \mathrm{G}$ or $25 \mathrm{G}$ ) were placed only once in the joint during the procedures. They reported a potential needle placement safe zone, which had a lower incidence of damage; however, any needle placed in this location still created damage to the joint. The zone was described to be cranial to the medial collateral ligament (O'Brien and Martinez, 2009). Based on the size of a jig pin (3 mm diameter) recommended for TPLO, it is obvious that intra-articular pin placement could cause much more damage when compared to a $20 \mathrm{G}$ needle $(0.9 \mathrm{~mm}$ diameter).

A study describing potential complications related to TPLO found that $1 \%$ of dogs had inadvertent intraarticular placement of jig pins (Priddy et al., 2003), which is lower than what was identified in our study. The discrepancy between the two studies may be due to the inclusion criteria (such as performing TPLO without a jig), incomplete details about radiographic findings in the medical records, and/or differences in methodology for evaluating radiographs between studies. The presence of intra-articular jig placement was not evaluated clinically in these previous studies or in our study and, therefore, it remains uncertain whether or not intra-articular jig pin placement will have an impact clinically. There is certainly evidence to suggest a compromise to the stifle in this manner can be clinically relevant. Progressive cartilage damage consistent with osteoarthritis was reported in a rabbit that had two holes drilled into a non-weight bearing region of their femur (Huebner et al., 2013). The rabbits were euthanized in subgroups at different times (up to 52 weeks), and stifles were evaluated grossly and histopathologically. Intra-articular cartilage damage with osteoarthritis was attributed to the presence of bone tunnels (Huebner et al., 2013). Therefore, it is likely that inadvertent intra-articular jig pin placement leads to progressive cartilage degeneration. Meniscal and cartilage damage due to temporary intra-articular pin placement may be potential causes for postoperative lameness after TPLO, and further investigation is needed to determine the clinical impact of these complications.

During our analysis, we found that the lateral tibial plateau may have been at higher risk for intra-articular damage based on the trajectory of the jig pin. Although anatomic variants of the tibial plateau exist between breeds (Ocal et al., 2013), in general, the lateral tibial condyle surface has a steeper slope than the medial tibial condyle (Sabanci and Ocal, 2014). The difference between plateau anatomy may lead to intraarticular jig pin placement in the lateral compartment, even if the pin is not entering the medial joint space, which happened when the jig pins were inserted too proximally. Another factor that led to intra-articular pin insertion in the lateral plateau was the angulation of the jig pin proximally. In both cases, the pins were inserted in the lateral plateau due to long drive distance within the tibia. The medial group intra-articular jig placement, however, occurred due to the insertion of the pin less than $2 \mathrm{~mm}$ from the medial plateau with or without proximal angulation of the pin.

Based on our study, a few recommendations should be followed when inserting the proximal jig pin to avoid intra-articular jig pin placement. The medial to lateral width of the tibial plateau should be measured, and the proximal jig pin inserted up to half of this measurement. In addition, the jig pin should be placed 3-4 $\mathrm{mm}$ distal to the articular surface, immediately caudal to the medial collateral ligament. Finally, the angle of the jig pin relative to the mechanical axis of the tibia should be assessed, and it should be perpendicular to the pin. We also evaluated whether a high TPA would increase the risk of intra-articular jig pin placement based on the fact that the caudal aspect of the joint line may be lower in dogs with higher TPA. However, this study did not support our hypothesis that TPA $>30^{\circ}$ would increase the likelihood of intra-articular jig pin placement. We believe that our hypothesis was rejected because we 
tend to place the jig pin slightly under the caudal aspect of the medial collateral ligament, which is cranial to regions where the lateral tibial condyle is lower than the medial tibial condyle.

Our hypothesis that the inadvertent intra-articular jig pin placement was related to less experienced surgeons was also rejected. It is important to point out that residents were performing surgeries under the guidance of the attending board-certified surgeon. In addition, the experience level of the surgeon was not assessed. There are several limitations to this study. The main limitation was the absence of assessment of the postoperative clinical and radiographic follow-up of this study; the clinical impact of our findings could not be quantified. Details about the physical examination, history, surgical report, and complications were not included in our study. Furthermore, the potential placement of the jig pin proximal to the tibial plateau might have occurred, which should not be revealed radiographically. Therefore, it is possible that intraarticular jig pin placement may be under-estimated. Another limitation is the potential lack of accuracy regarding the primary surgeon experience level.

In conclusion, intra-articular placement of the jig pins was uncommon but was identified in over 5\% of our cases. Our findings serve as a reminder to take precaution when placing the proximal jig pin during TPLO and have provided guidelines for evaluating proximal jig pin placement on postoperative radiographs.

\section{Acknowledgment}

The authors would like to thank Edson Oliveira, Jr., for helping in converting all the collected data in Excel tables and teaching the world of Pivot Tables.

\section{Funding}

The authors received no specific grant from any funding agency in the public, commercial, or not-forprofit sectors for the preparation of this project.

\section{Conflict of interest}

The authors do not have any potential conflicts of interest to declare.

\section{Author's contribution}

Jacqueline V. J. Cavalcanti performed the data collection and analysis, and wrote the manuscript. Stanley E. Kim performed the study design and supervised the study. James Colee performed statistical analysis. All authors revised and approved the manuscript before submission.

\section{References}

Devine, T., Slocum, B., Boulay, J. and Gores, B. 2003. TPLO - surgical technique. In TPLO seminar course documents, Eugene, OR.

Dismukes, D.I., Tomlinson, J.L., Fox, D.B., Cook, J.L. and Witsberger, T.H. 2008. Radiographic measurement of canine tibial angles in the sagittal plane. Vet. Surg. 37, 300-305.

Duerr, F.M., Martin, K.W., Rishniw, M., Palmer, R.H. and Selmic, L.E. 2014. Treatment of canine cranial cruciate ligament disease. A survey of ACVS diplomates and primary care veterinarians. Vet. Comp. Orthop. Traumatol. 27, 478-483.

Fitzpatrick, N. and Solano, M.A. 2010. Predictive variables for complications after TPLO with stifle inspection by arthrotomy in 1000 consecutive dogs. Vet. Surg. 39, 460-474.

Huebner, K.D., Shrive, N.G. and Frank, C.B. 2013. New surgical model of post-traumatic osteoarthritis: Isolated intra-articular bone injury in the rabbit. Orthop. Res. 31, 914-920.

Kowaleski, M.P., Boudrieau, R.J. and Pozzi, A. 2012. Stifle joint. In veterinary surgery small animal, Eds., Tobias, K.M. and Johnston, S.A. St. Louis, MI: Elsevier, pp: 906-998.

O'Brien, C.S. and Martinez, S.A. 2009. Potential iatrogenic medial meniscal damage during tibial plateau leveling osteotomy. Vet. Surg. 38, 868-873.

Ocal, M.K., Sabanci, S.S. and Onar, V. 2013. Variation of tibial plateau geometry and cruciate ligament coordinates in six breeds dogs. Vet. Comp. Orthop. Traumatol. 26, 110-116.

Pacchiana, P.D., Morris, E., Gillings, S.L., Jessem, C.R. and Lipowitz, A.J. 2003. Surgical and postoperative complications associated with tibial plateau leveling osteotomy in dogs with cranial cruciate ligament rupture: 397 cases (1998-2001). J. Am.Vet. Med. Assoc. 222, 184-193.

Priddy, II, N.H., Tomlinson, J.L., Dodam, J.R. and Hornbostel, J.E. 2003. Complications with and owner assessment of the outcome of tibial plateau leveling osteotomy for treatment of cranial cruciate ligament rupture in dogs: 193 cases (1997-2001). J. Am. Vet. Med. Assoc. 222, 1726-1732.

Sabanci, S.S. and Ocal, M.K. 2014. Lateral and medial tibial plateau angles in normal dogs. An osteological study. Vet. Comp. Orthop. Traumatol. 27, 135-140.

Schmerbach, K.I., Boeltzig, C.K., Reif, U, Wieser, J.C., Keller, T. and Grevel, V. 2007. In vitro comparison of tibial plateau leveling with and without use of a tibial plateau leveling jig. Vet. Surg. 36, 156-163.

Slocum, B. and Slocum, T.D. 1993. Tibial plateau leveling osteotomy for repair of cranial cruciate ligament rupture in the canine. Vet. Clin. North Am. Small Anim. Pract. 23, 777-795.

Thieman, K.M., Tomlinson, J.L., Fox, D.B., Cook, C. and Cook, J.K. 2008. Effect of meniscal release on rate of subsequent meniscal tears and ownerassessed outcome in dogs with cruciate disease treated with tibial plateau leveling osteotomy. Vet. Surg. 35, 705-710. 